\title{
Age-related changes in collagen degeneration of the rotator cuff in an animal model
}

\author{
Kenji Murata $^{1}$, Naohiko Kanemura ${ }^{1,}$ *, Takanori Kokubun ${ }^{1}$, Tsutomu Fujino ${ }^{2}$, Junichi Matsumoto ${ }^{3}$, \\ Kenji Yasui $^{4}$, Kiyomi Takayanagi ${ }^{1}$ \\ ${ }^{1}$ Department of Physical therapy, School of health and Social Services, Saitama Prefectural University, Saitama, Japan \\ ${ }^{2}$ Department of Rehabilitation, Urawa Orthopedics Clinic, Saitama, Japan \\ ${ }^{3}$ Department of Rehabilitation, Kasukabe Chuo general Hospital, Saitama, Japan \\ ${ }^{4}$ Department of Orthopedics, Tokyo Women's Medical University, Tokyo, Japan
}

\section{Email address:}

muraken720@gmail.com (Murata K.), kanemura-naohiko@spu.ac.jp (Kanemura N.),fbtribe20@gmail.com (Fujino T.), kokubun-takanori@spu.ac.jp (Kokubun T.),jun1-matsu@hotmail.co.jp (Matsumoto J.), kej.yasui@gamil.com (Yasui K.), takayanagi-kiyomi@spu.ac.jp (Takayanagi K.)

\section{To cite this article:}

Kenji Murata, Naohiko Kanemura, Takanori Kokubun, Tsutomu Fujino, Junichi Matsumoto, Kenji Yasui, Kiyomi Takayanagi. Age-Related Changes in Collagen Degeneration of the Rotator Cuff in an Animal Model. American Journal of Biomedical and Life Sciences.

Vol. 2, No. 6, 2014, pp. 156-162. doi: 10.11648/j.ajbls.20140206.14

\begin{abstract}
Rotator cuff tears are a common injury in the elderly and often impose limitations on the range of motion and cause pain. The cause of rotator cuff tearing is unknown, and degeneration of the supraspinatus tendon with age is poorly understood.In the present study, we examined collagen degeneration of the supraspinatus tendon in the rat shoulder joint. Using rats of different age (10 weeks: young, 6 months: adult, and 2 years: old), we examined the supraspinatus tendon by immunofluorescence staining (collagen types I and III), assessed the amount of collagen and mRNA expression for collagen type 1A1, 3A1 (COL1A1, COL3A1), matrix metalloproteinase 2,9 (MMP-2, -9).After immunofluorescence staining, the layer structure of tendon to bone attachment was apparent in the young. The amount of collagen decreased in the old group. In addition the mRNA expression of Collal and Col3al was significantly reduced in the old group.On the one hand, the old groups showed higher mRNA levels of $M M P-2$ and $M M P-9$ than the young group. The ability to synthesize collagen decreases during aging and the amount of total collagen that is present in the supraspinatus tendon is decreased. These changes may contribute to tearing/breakage of the supraspinatus tendon.
\end{abstract}

Keywords: Supraspinatus Tendon, Aging, Collagen

\section{Introduction}

Rotator cuff tears are a common cause of shoulder pain, poor shoulder function, and activity of daily living disability in the elderly population [1-3]. The rotator cuff is composed of four tendons and muscles: supraspinatus, infraspinatus, teres minor, and subscapularis. The role of the rotator cuff is to connect the scapula and the upper humerus. These muscles are important in shoulder movements and in maintaining stability of the glenohumeral joint. Rotator cuff tears often occur because the tendons have been weakened through the natural aging process or other degeneration.

The etiology of rotator cuff tears is multifactorial: several studies have reported that both extrinsic factors and intrinsic factors are involved in the development of rotator cuff tear
[4,5]. For example, the extrinsic factors include increased subacromialloading [6], overload, and muscle imbalance [7], whereas intrinsic factors arise from age-related degeneration $[8,9]$. Nowadays, it is generally believed that age-related degenerative changes in the rotator cuff tendon are an important intrinsic factor, and previous studies have shown that the rotator cuff can tear naturally with age [8-10]. However the process of a break in the rotator cuff is poorly understood.

The extracellular matrix of the supraspinatus tendon is largely composed of type I collagen, type III collagen and elastin. Collagen gives strength to the tendon and elastin gives viscoelasticity, however the denaturation of collagen is 
associated with rotator cuff tears. Thus, although the collagen fibers are an integral component of the structure of the tendon, degeneration of the collagen of the supraspinatus tendon involves fatty infiltration [11-13], glycation[14], and calcification [15]. Matrix metalloproteinases (MMPs) are a family of extracellular matrix degrading enzymes. MMPs are important factors of tendon healing. MMP-2 and MMP-9, a member of the gelatinase family of MMPs, mediates leukocyte migration during inflammation. Activities of MMP-2 and MMP-9 in tendon also increase with age. However, the influence of aging is not considered in the analysis of supraspinatus tendon damage. The precise cause of rotator cuff injury has yet to be identified because it is difficult to biopsy healthy tissue.

The purpose of this study is to clarify the degenerative process due to aging of the supraspinatus tendon. We followed these steps: (1) to measure expression of collagen genes in the supraspinatus tendon by means of quantitative real-time polymerase chain reaction (PCR), (2) to compare these changes using histological and immunofluorescence staining, and (3) to quantify enzyme-soluble collagen using the collagenase technique. The hypothesis of this study is that collagen of the supraspinatus tendon is denatured with age, and the amount of soluble collagen is reduced by fibrosis associated with aging.

\section{Materials and Methods}

\subsection{Animals}

All experiments were approved by the Animal Research Committee of Saitama Prefectural University (Saitama, Japan). In the present study, 30 Wistar rats (Clea Japan, Tokyo, Japan) were used at different ages (10weeks: young, 6months: adult, and 24 months: old). All groups were housed in a room with a temperature of $23 \pm 1^{\circ} \mathrm{C}$ and 12/12-h light/dark cycle. None of the groupswas limited in terms of movement within the cage.

\subsection{Histological Observations}

After experiments, animals were anesthetized using pentobarbital $(50 \mathrm{~mL} / \mathrm{kg})$. We collected the rat shoulder joint and included all tuberosity. The specimens were fixed in $4 \%$ paraformaldehyde solution in phosphate buffered saline (PBS) for $24 \mathrm{~h}$ at $4{ }^{\circ} \mathrm{C}$. Following which, the samples were decalcified with $10 \%$ EDTA (Sigma-Aldrich, Japan) with $2.42 \%$ (wt/vol) tris-hydroxymethyl-aminomethane (Fisher Scientific, USA) at $\mathrm{pH} 7.3$ for approximately 45 days. The decalcifying solution was renewed twice a week. After the completion of decalcification, the joint samples were immersed in a sucrose solution of different concentrations (10\%: $4 \mathrm{~h}, 15 \%$ : $4 \mathrm{~h}$, and 20\%: 8h). Thereafter, they were embedded in OCTcompound (Sakura Finetek, Torrance, CA), and $14-\mu \mathrm{m}$ sections were cut on a Leica CM $3050 \mathrm{~S}$ cryostat(Leica Microsystems AG, Wetzlar, Germany). These sections were stained with toluidine blue (TB) and hematoxylin and eosin (H\&E) to examine the attachment of the tendon and bone.

\subsection{Immunofluorescence Assays}

We assessed the age-related collagen changes using immunohistological staining of the attachment of the tendon and bone. Sections were air-dried for $30 \mathrm{~min}$ and washed 3 times for 5 minwith $0.01 \mathrm{M}$ PBS ( $\mathrm{pH} 7.4$ ). Endogenous peroxidase was inactivated by incubating sections in $0.3 \%$ $\mathrm{H}_{2} \mathrm{O}_{2}$ for $30 \mathrm{~min}$. The excess endogenous peroxidase was removed by washing 3 times for $5 \mathrm{~min}$ in PBS. For immunofluorescence assays, the sections were incubated with a rabbit polyclonal anti-collagen typeI-antibody(dilution 1:500; Abcam, Cambridge, USA), anti-collagen type II-antibody (dilution 1:500; Abcam, Cambridge, USA), and anti-collagen type III-antibody(dilution 1:500; Abcam, Cambridge, USA)overnight at $4^{\circ} \mathrm{C}$. The next day, the sections were visualized using aDyLight-488 conjugated goat anti-rabbit IgG secondary antibody (dilution 1:250; Thermo, Rockford, USA). Subcellular localization of collagen was examined under a fluorescence microscope.

\subsection{Real-Time RT-PCR}

Supraspinatus tendons were trimmed to $30 \mathrm{mg}$, and total RNA was extracted using the RNeasy Fibrous Tissue Mini Kit (Qiagen, Valencia, CA) according to the manufacturer's instructions. Synthesis of the cDNA was conducted using a high-capacity cDNA reverse transcription kit (Applied Biosystems, USA). Real-time PCR was performed on a StepOne-Plus real-time system (Applied Biosystems, USA) using $1 \mu \mathrm{L}$ of cDNA in the presence of TaqMan primers (Grand Island, New York, USA). The relative expression of each gene was normalized to $\beta$-actin to obtain a relative expression level. All reagents required for real-timePCR methods came from the TaqMan primer kit (Applied Biosystems, USA). The primersused were as follows: Colla1 (Rn01463848), Col2al (Rn1637087), Col3al (Rn0143681), $T G F-\beta\left(\mathrm{Rn} 01440676 \_\mathrm{m} 1\right), M M P-2 \quad\left(\mathrm{Rn} 01538171 \_\mathrm{m} 1\right)$ and $M M P-9(\mathrm{Rn} 0057916 \overline{2} \mathrm{~m} 1)$.

\subsection{Quantification of Collagen}

The methods of novel fluorescence (FL) for N-terminal glycine-containing peptides had been developed using 3,4-dihydroxyphenylacetic acid (DHPAA)[16]. N-terminal peptides were obtained by digesting the tendon with collagenase. After collecting the supraspinatus tendon, the dry weight was measured. Collagenase solution $0.01 \%(250 \mu \mathrm{L})$ in borate buffer was prepared (Collagenase, Wako, Japan) to dissolve the tissue $\left(37^{\circ} \mathrm{C}, 1 \mathrm{~h}\right)$. Thereafter, $250 \mu \mathrm{L}$ of $0.75 \mathrm{mM}$

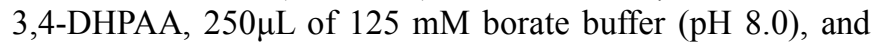
$250 \mu \mathrm{L}$ of $1.25 \mathrm{mM}$ sodium periodate were successively added. The solution $(1000 \mu \mathrm{L})$ was incubated for $10 \mathrm{~min}$ at $37^{\circ} \mathrm{C}$. The excitation and emission maximum wavelengths were set at $370 \mathrm{~nm}$ and $465 \mathrm{~nm}$ respectively. Enzyme-soluble collagen content was calculated using the standard curve. Collagen peptide solutions of different concentrations were prepared ( $0.0001 \%$ to $0.01 \%$ collagen concentration). These solutions were used to construct the standard curve using the logarithm of the dilution factor. 


\subsection{Statistics}

The values among the 3 groups were compared using one-way analysis of variance (ANOVA). To compare the differences of amount of collagen between middle-aged and old rats, the Mann-Whitney test was used. Statistical analyses were performed using SPSS ver. 21 for Windows (International Business Machines Corporation, USA). Any p-values $<0.05$ were assumed to mean statistical significance.

\section{Results}

\subsection{Histological and Immunohistochemical Observations}

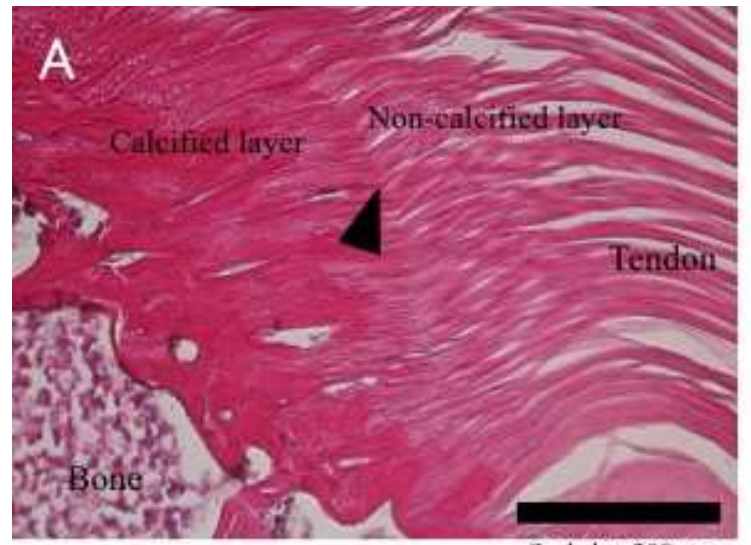

Scale bar $200 \mu \mathrm{m}$
Figure 1 and Figure 2 shows the typical histopathological changes in the supraspinatus tendon and the adjacent bone. In H\&E staining, the un-calcified cartilage layer and calcified cartilage layer were observed at the boundary tide-mark (fig.1A, B). In TB staining, the fibrocartilage layer was increasingly stained with age (fig.2A,B and C).Figure2D,E and $\mathrm{F}$ showed the staining of the articular cartilage of the same shoulder sample, but there was no difference by aging.

Immunostaining for type I collagen showed specific staining patterns for each age group of the enthesis: the fluorescence at enthesis was decreased in old rats, and adult $\operatorname{rats}($ fig. $3 \mathrm{~A}, \mathrm{~B}$ and $\mathrm{C}$ ). On the other hand, the staining patterns of type III collagen were observed to old rats (fig.3D, E and F).

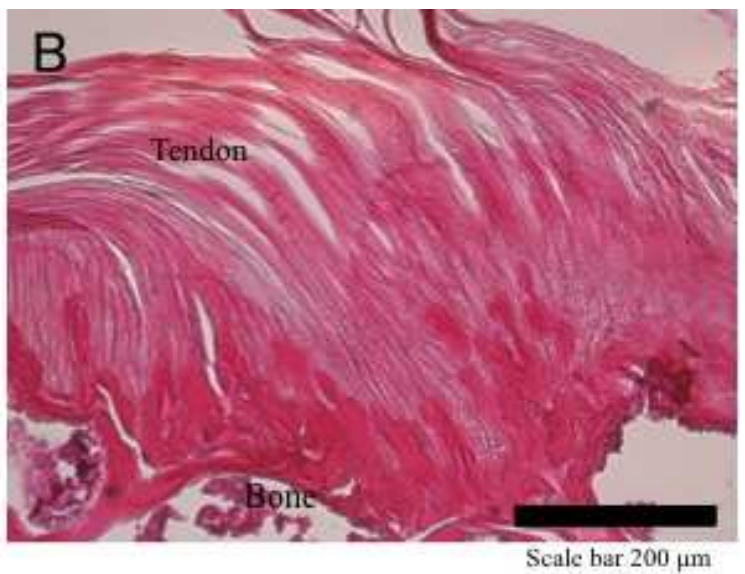

Figure 1. This figure shows the tendon-bone attachment region. (A) Young group. Arrowhead indicate tidemark. (Between the non-calcified cartilage layer and calcified cartilage layer)(B) Old group. Tidemark is unclear. Scale bar: $200 \mu m$.
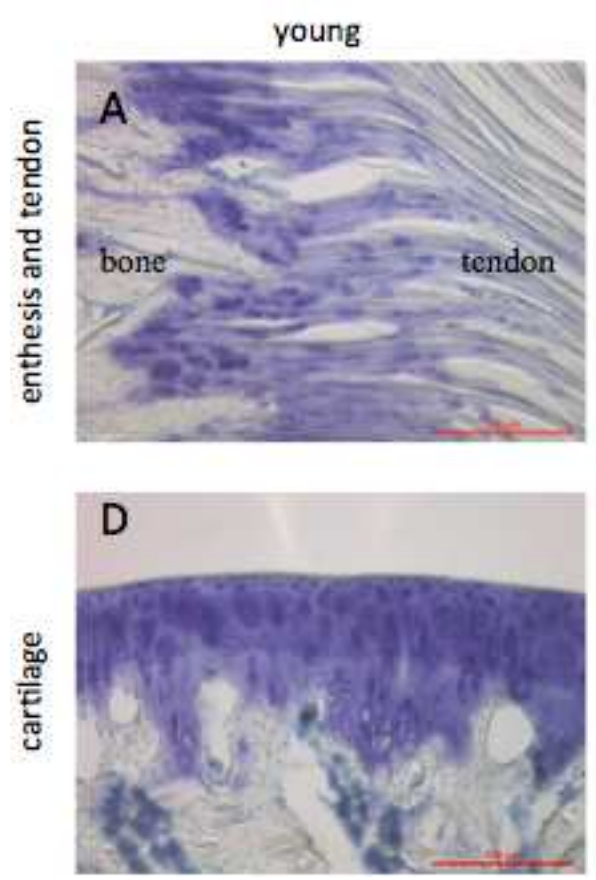

adult
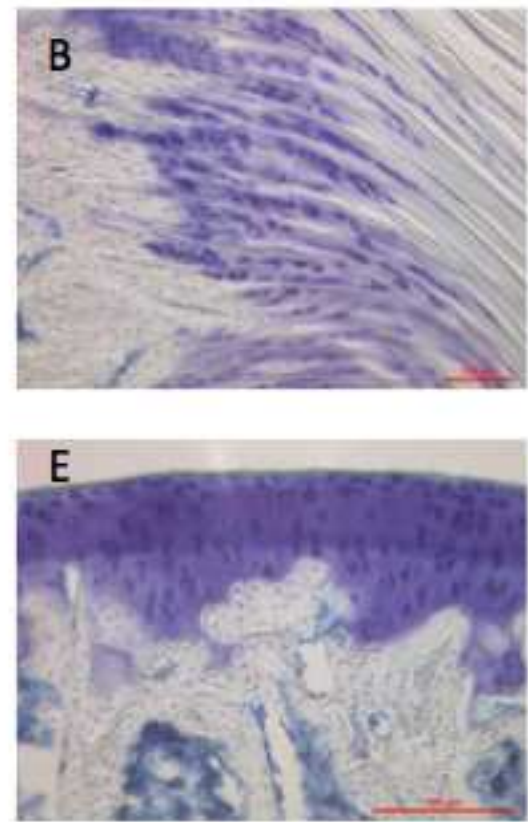

old
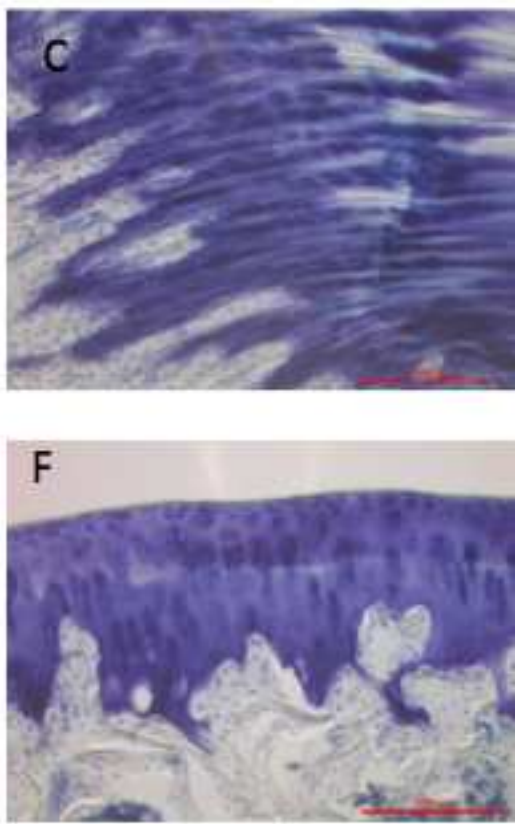

Figure 2. Staining with toluidine blue.Enthesis of supraspinatus tendon $(A-C)$, articular cartilage of humeral head (D-F), the young group (A, D), the adults group $(B, E)$ and the old group $(C, F)$. Scale bar: $100 \mu m$. 

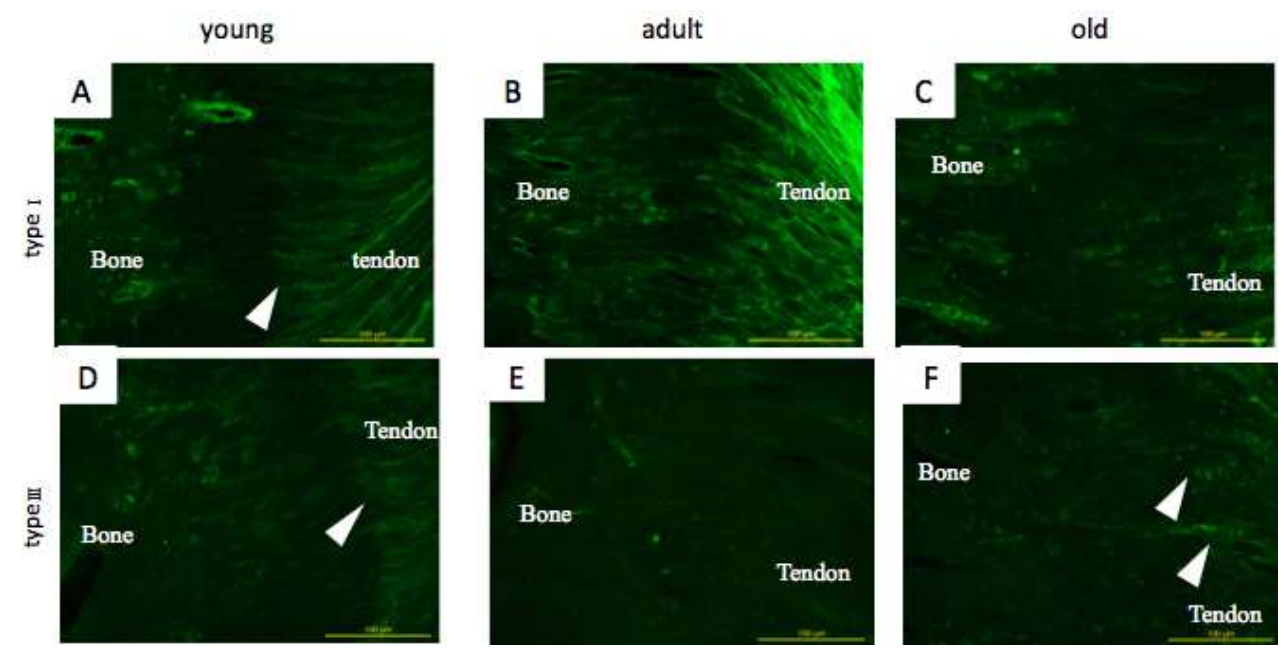

Figure 3. Age-related changes of collagen type by immunofluorescence staining.Immunofluorescence staining images of type I collagen (A, B, C) and type III collagen $(D, E, F)$. The Arrowhead of $(A, D)$ : the layer structure of tendon to bone attachment was apparent in the young. The Arrowhead of $(F)$ : type III collagen

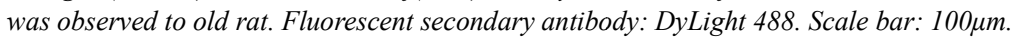

\subsection{Real-Time PCR}

The results showed that the mRNA expression of Collaland Col3alwas downregulated with aging $(\mathrm{p}<0.01)$ (fig.4A,B). On the other hand, the mRNA expression level of Col2al in the old group was higher than that in the young and adult group. The adult and old groups showed higher mRNA levels of $M M P-2$ and $M M P-9$ than the young group $(\mathrm{p}<0.01)$ (fig.5B, C). However, there was no difference in the mRNA levels of $T G F$ - $\beta$ among three groups (fig.5A).
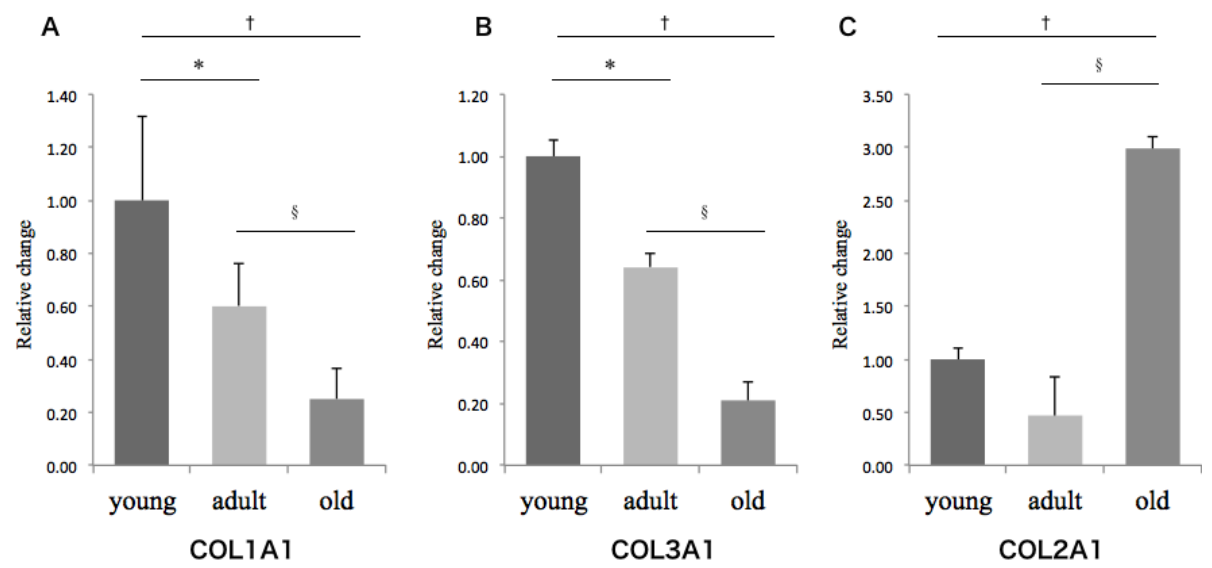

Figure 4. ThemRNA expression levels were analyzed using the $\triangle \triangle C T$ method, and we calculated the relative value in one of the young rats. Collal mRNA expression levels were significantly decreased with age $(A)$. There was also a significant amount of Col3al mRNA, which decreased with age (B). However, Col2al mRNA expression increased with age (C). The data are shown as mean $\pm S E$; *young vs. adult, ${ }^{\dagger}$ young vs. old, ${ }^{\xi}$ adult vs. old; $p<0.05$.
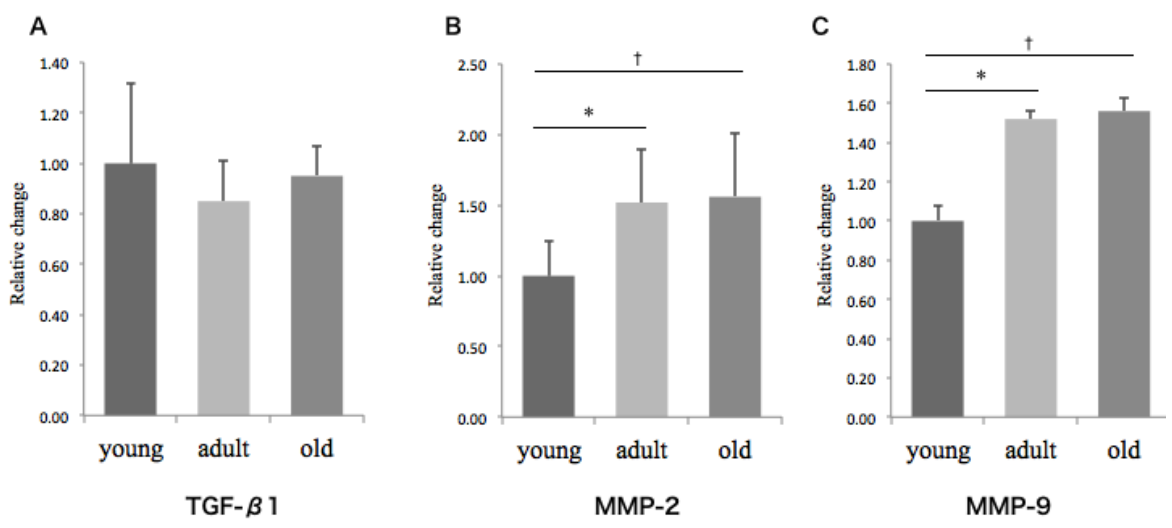

Figure 5. MMP-2 and MMP-9 $m R N A$ expression levels were significantly increased with age $(B, C)$. There was no significant amount of TGF- $\beta m R N A(A)$. The data are shown as mean $\pm S E ; *^{*}$ young vs. adult, ${ }^{\dagger}$ young $v$ s. old $; p<0.05$. 
A

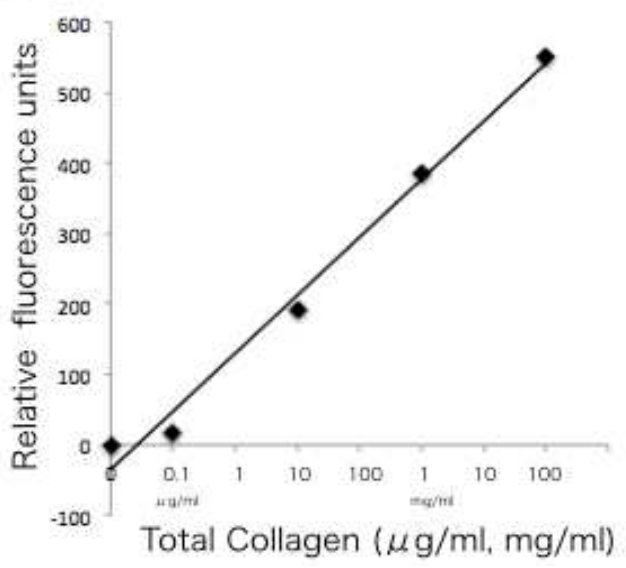

B

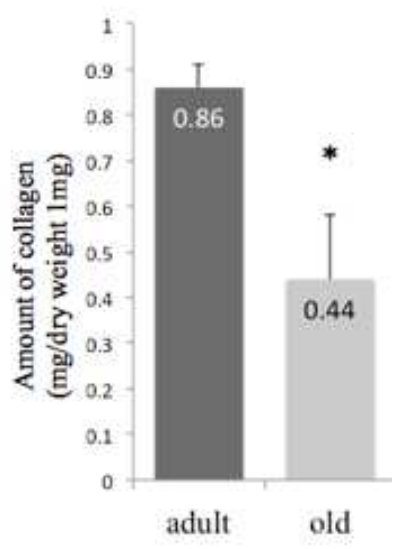

Figure 6. Construction of the standard curve (A). We made serial collagen dilutions (standard solutions), and measured fluorescence of each solution (B). * $<<$ 0.05 .

\subsection{Quantification of Collagen}

Using a calibration curve, we quantified collagen of the supraspinatus tendon (fig.6A). The amount of this type of collagen was significantly decreased in aging rats compared with rats at 6 months of age (fig.6B).

\section{Discussion}

Tendon has degenerated as a result of overuse or age-related degenerative changes. When degeneration of the tendon and enthesis occurs in inflammation, it is referred to as "tendinopathy". Common painful tendinopathies include patellar tendinopathy, Achilles tendinopathy and rotator cuff tendinopathy. Above all, rotator cuff injury is one of the most common causes of shoulder pain. In the present study, we assessed the histological and biochemical degenerative changes of collagen in the supraspinatus tendon.

The results of this study indicate that mRNA expression of Collal and Col3al was decreased during aging. These findings are also supported by the observed structure of the tendon and by immunofluorescence staining. The results clearly are affected by aging in the same environment. Type I collagen is the main component of the tendon along with elastin, and fibrocartilage of enthesis also consists of type I collagen. Because collagen is a protein undergoing slow metabolism, it is necessary to prevent modifications to maintain the function of the collagen. However, the process of aging is intrinsic process that should be considered innate to our genetic design. Many change occur at the cellular level in an aging organization. Importantly, there is a capability to attempt to recover aging changes - maintain the ability to synthesize new collagen to prevent joint diseases and aging. However, the capacity of collagen synthetic has reduced by aging in this study, and the balance between collagen synthesis and degradation in the tendon might lose by the aging.

TGF-ßand MMP are involved in remodeling of the extracellular matrix of tendons. In particular, the presence of MMP-2 and MMP-9 are typical signs of inflammatory. The expression of MMP-2 increased in Achilles tendinopathy [17].
High glucose concentrations increased the expression of MMP-9. In the aging tendon, advanced glycation end products (AGEs), resulting from the exposure of proteins to high levels of sugar accumulate in the supraspinatus tendon. In the present study, the adult and old groups showed higher mRNA levels of MMP-2 and MMP-9 than the young group.

MMP-2 and MMP-9 are produced by inflammatory cells, and the age-related molecular of the tendon in this study could contribute to the tearing of the supraspinatus tendon.

However, the 4-layer structure of the tendon-bone attachment region was not observed in the histological and immunofluorescence staining experiments in aging rats. Among them, the some signs of damage were observed. First, In the structural organization observed, there is a difference in the fluorescence signal by age in the attachment of the supraspinatus tendon to the bone. The layer structure in type I collagen was apparent in middle-aged rats but not in old rats. In general, the tendon-bone attachment region is composed of 4 layers: the tendon, non-calcified cartilage layer, calcified cartilage layer, and bone [18]. Such layered attachment is contribute in the maintenance of mechanical strength, and indicate that the tendon-bone attachment region exhibits normal functioning. The amount of type 3collagen has decreased in the tendon-bone attachment region with age in previous research[19]. Supraspinatus tendon was also similar results.

Secondary, in the old group, type III collagen was presented in the tendon layers. Type III collagen is often expressed during remodeling of the tendon that is impaired [20]. A study on pathological changes in the human Achilles tendon showed that the mRNAs levels of type I and type III collagen are significantly higher in tendons with chronic pain or spontaneous tearing/breakage than in healthy tendons [21]. Furthermore, mRNA and protein expression of type III collagen has increased at the healing ligament in our laboratory research. In the present study, age alone may not be sufficient to damage the supraspinatus tendon. However, the observation of type III collagen is likely to be a sign of age-related collagen degradation. 
Finally, our results showed collagen content of the supraspinatus tendon was reduced by aging. These are the result of an imbalance of the synthesis and degradation of collagen: degraded by MMP-2 and -9 has been promoted, and the synthesis of collagen has been inhibited. Collagen maintains the mechanical strength via crosslinking, and the balance of synthesis and degradation maintains the collagen tissue. Aging reduces homeostasis, and it might be a cause of tendon rupture. However, the expression of TGF- $\beta$ mRNA was no difference. The results might showed that collagen synthesis in the aging tendon is induced in the other factors.

\section{Conclusions}

In conclusion, fibroblast proliferation and tearing/breakage of the tendon were not observed in aging rats. This finding indicates that instead of degeneration of collagen by injury we see degeneration that is age related. These results may help to elucidate the mechanism of tearing/breakage of the supraspinatus tendon associated with aging. Several studies have reported that this mechanism may be due to mechanical stress on rotator cuff and the resulting injury[22,23].In contrast, we believe that mechanical stress is not the primary cause, and the rotator cuff injury may be biological, i.e., age-related. Thus, development of rotator cuff injury is linked to the interplay of chemical and mechanical factors.

\section{Acknowledgments}

This research was partially supported by Japanese Society for the Promotion of Rehabilitation (2012).

\section{References}

[1] Mall N A, Kim H M, Keener J D, StegerMay K, Teefey S A, MiddletonW D, Yamaguchi K, "Symptomatic Progression of Asymptomatic Rotator Cuff Tears. A Prospective Study of Clinical and SonographicVariables,"JBoneJointSurg Am, 92, 2623-2633, 2010.

[2] Milgrom C, Schaffler M, Gilbert S, Van Holsbeeck M, "Rotator cuff changes in asymptomatic adults. The effect of age, hand dominance and gender,"JBoneJointSurg Br, 77(2), 296-298, 1995.

[3] Tempelhof S, Rupp S, Seil R, "Age-related prevalence of rotator cuff tears in asymptomatic shoulders"J Shoulder Elbow Surg 8, 296-299, 1999.

[4] Carpenter J E, Flanagan C L, Thomopoulos S, Yian E H, Soslowsky L J, "The effects of overuse combined with intrinsic or extrinsic alterations in an animal model of rotator cuff tendinosis,"Am J Sports Med, 26, 801-807, 1998.

[5] Kaux JF, Forthomme B, Goff CL, Crielaard JM, Croisier JL., "Current opinions on tendinopathy,"J Sports Sci Med,10, 238-253, 2011.

[6] Michener L A, McClure P W, Karduna A R, “Anatomical and biomechanical mechanisms of subacromial impingement syndrome,"Clinical biomechanics, 18, 369-379, 2003.
[7] Reuther K E, Thomas S J, Tucker J J, Sarver J J, Gray C F, Rooney S I, Soslowsky L J, "Disruption of the anterior-posterior rotator cuff force balance alters joint function and leads to joint damage in a rat model,"JOrthop Res,232, 638-44, 2014.

[8] Sano H, Ishii H, Trudel G, Uhthoff H K, "Histologic evidence of degeneration at the insertion of 3 rotator cuff tendons: a comparative study with human cadaveric shoulders,"Journal of Shoulder and Elbow Surgery, 8, 574-579, 1999.

[9] Soslowsky L J, Thomopoulos S, Tun S, Flanagan C L, Keefer C C, Mostow J, Carpenter J E, "Overuse activity injures the supraspinatus tendon in an animal model: A histologic and biomechanical study,"J Shoulder Elbow Surg, 9, 79-84, 2000.

[10] Yamaguchi K, Tetro A M, Blam O, Evanoff B A, Teefey S A, Middleton W D, "Natural history of asymptomatic rotator cuff tears: a longitudinal analysis of asymptomatic tears detected sonographically,"J Shoulder Elbow Surg, 10, 199-203, 2001.

[11] Gerber C, Meyer D C, Schneeberger A G, Hoppeler H, Von Rechenberg B, "Effect of tendon release and delayed repair on the structure of the muscles of the rotator cuff: an experimental study in sheep,"J Bone Joint Surg Am, 86, 1973-1982, 2004.

[12] Itoigawa $\mathrm{Y}$, Kishimoto N, Sano $\mathrm{H}$, Kaneko K, Itoi E, "Molecular mechanism of fatty degeneration in rotator cuff muscle with tendon rupture,"JOrthop Res, 29, 861-866, 2011.

[13] Melis B, DeFranco M J, Chuinard C, Walch G, "Natural history of fatty infiltration and atrophy of the supraspinatus muscle in rotator cuff tears,"ClinOrthopRelat Res, 468, 1498-1505, 2010.

[14] Bedi A, Fox A J, Harris P E, Deng X H, Ying L, Warren R F, Rodeo S A, "Diabetes mellitus impairs tendon-bone healing after rotator cuff repair,"J Shoulder Elbow Surg, 19, 978-988, 2011.

[15] Chard M D, Cawston T E, Riley G P, Gresham G A, Hazleman B L, "Rotator cuff degeneration and lateral epicondylitis: a comparative histological study,"Ann Rheum Dis, 53, 30-34, 1994.

[16] Yasmin H, Shibata T, Rahman M S, Kabashima T, Kai M, "Selective and sensitive determination of peptides using 3,4-dihydroxyphenylacetic acid as a fluorogenic reagent," Anal ChimActa. 721, 162-166, 2012.

[17] Alfredson H, Lorentzon M, Backman S, Backman A, Lerner $\mathrm{UH}$, "cDNA-arrays and real-time quantitative PCR techniques in the investigation of chronic Achilles tendinosis,"J Orthop Res. 21:970-975, 2003.

[18] Benjamin M, Ralphs J R, "Fibrocartilage in tendons and ligaments-an adaptation to compressive load,"J anatomy, 193, 481-494, 1998.

[19] Benjamin M, McGonagle D, "The anatomical basis for disease localisation in seronegativespondyloarthropathy at entheses and related sites,"Journal of Anatomy, 199, 503-526, 2001.

[20] Eriksen H A, Pajala A, Leppilahti J,Risteli J, "Increased content of type III collagen at the rupture site of human Achilles tendon," J Orthop Res, 20, 1352-1357, 2002.

[21] Hirose K, Kondo S, Choi H R, Mishima S, Iwata H, Ishiguro N, "Spontaneous healing process of a supraspinatus tendon tear in rabbits," Archives of orthopaedic and trauma surgery, 124, 374-377, 2004. 
[22] Liem D, Buschmann VE, Schmidt C, Gosheger G, Vogler T, Schulte TL, Balke M, "The prevalence of rotator cuff tears: is the contralateral shoulder at risk?" Am J Sports Med, 42, 826-30, 2014
[23] Moor B K, Wieser K, Slankamenac K, Gerber C, Bouaicha S, "Relationship of individual scapular anatomy and degenerative rotator cuff tears,"J Shoulder Elbow Surg, 23, 536-541, 2014. 